\section{Manifestaciones estomatológicas en pacientes VIH/SIDA}

\section{Estomatologic manifestations in HIV/AIDS patients}

\section{Resumen}

El presente estudio se realizó en 128 pacientes infectados por el virus de inmunodeficiencia humana, en el Hospital Guillermo Almenara Irigoyen, con la finalidad de encontrar la frecuencia de lesiones orales y la ubicación de éstas en los pacientes VIH/SIDA de acuerdo a la edad y sexo; además establecer la relación existente entre la cifra de linfocitos CD4 con las lesiones detectadas. La muestra fue tomada de manera probabilística; el método utilizado para la recolección de datos fue la observación y la información obtenida se registro en una ficha previamente diseñada. De la muestra 74 pertenecían al sexo masculino y 57 al femenino y sólo el $57,8 \%$ presentaban lesiones estomatológicas. En relación a la edad, el mayor número de pacientes se encontraba ubicado en el grupo de 26 a 35 años (43,8\%). Las lesiones que se presentaron con mayor frecuencia fueron: la candidiasis pseudomembranosa $(21,9 \%)$ ubicándose principalmente en lengua, paladar duro y carrillo, úlceras aftosas $(14,1 \%)$ ubicándose principalmente en carrillo, lengua y labio, la candidiasis eritematosa $(10,1 \%)$ principalmente en paladar blando, queilitis angular $(10,1 \%)$ y la leucoplasia vellosa $(7 \%)$. Existe una relación significativa entre la presencia de candidiasis pseudomembranosa y la leucoplasia oral vellosa con niveles de CD4 menores de $200 \mathrm{cel} / \mathrm{mm} 3(\mathrm{p}<0,05)$.

\section{Abstract}

The study was carried out at Hospital Nacional Guillermo Almenara Irigoyen, on 128 patients infected by the human inmunodefense virus. The aim of the investigation was to study the frecuency, and location of oral lesions, in relation with age and sex, also to establish the relationship between $\mathrm{CD} 4$ cells counts with oral lesions detected. The sample were selected in a probabilistic way; the data was recorded in a special designed format. 74 subjects of the sample were male and 57 female. Oral lesions were found in $57,8 \%$ and $43,8 \%$ of the infected patients were between 26 and 35 ages. Pseudomembranous candidiasis was the most common lesion detected in $21,9 \%$ of patients (tongue, soft palate and cheeks), ulcers $14,1 \%$ (cheek, tongue and lips), erytematous candidiasis in 10,1\% (soft palate), angular queilitis in $10,1 \%$ and oral hairy leukoplakia in $7 \%$. Pseudomembranous candidiasis and oral hairy leukoplakia are clinical indicators of immunologic deterioration ( CD4 $200 \mathrm{ce} / \mathrm{mm} 3$ ).

\section{Introducción}

Los primeros reportes datan de 1978 (Canadá) y 1980 (Estados Unidos), años en que se documenta la deficiencia inmune asociada a enfermedades oportunistas como la neumonía por Pneumocistis carinii en un grupo de varones homosexuales. El seguimiento epidemiológico mostró la presencia de una nueva infección producida por un virus desconocido, originada en el África y que hasta ese momento había afectado a individuos con antecedentes de promiscuidad sexual, preferentemente con participación en relaciones homosexuales.

En 1983, Montagnier lo identifica, se trata de un nuevo retrovirus humano denominado virus de la inmunodeficiencia humana tipo1 (HIV-1) no oncogénico que se ha diseminado rápidamente por todo el mundo durante los últimos años, dando lugar a una pandemia de consecuencias imprevisibles. ${ }^{1}$

Las manifestaciones estomatológicas que se presentan en la infección por VIH-SIDA son diversas. Lifson ${ }^{2}$ en 1993 nos manifiesta que la presencia de ciertas lesiones orales sugiere que un individuo puede estar infectado por el VIH y otras lesiones pueden ayudar a indicar el pronóstico para la progresión del SIDA.

Greenspan Deborah ${ }^{3}$ en 1994, menciona que las manifestaciones de la infección por VIH son comunes e incluye lesiones orales nuevas $\mathrm{y}$ diferentes presentaciones de enfermedades oportunistas ya conocidas. El reconocimiento requiere de una cuidadosa historia clínica y revisión de la cavidad oral; este reconocimien- to puede cambiar la clasificación del estadío en la infección por VIH.

Schmidt-Westhausen et al. ${ }^{4}$ en 1997 describieron lesiones en mujeres HIV infectadas y determinaron la asociación de las lesiones orales con el nivel de inmunosupresión y la ruta de transmisión. La candidiasis se presenta más en casos de conteo bajo de CD4; mientras que no hay relación entre el nivel de inmunosupresión y la leucoplasia vellosa.

Greespan J.S. ${ }^{5}$ en 1997 nos describe a la candidiasis pseudomembranosa y a la leucoplasia pacientes HIV y SIDA; con una correlación entre la caída de CD4 y la progresión de la enfermedad, Pacientes con lesiones como candidiasis o leucoplaquia desarrollan más rápidamente la enfermedad que aquellos individuos que no las presentan; siendo las lesiones orales un indicador de la progresión de la enfermedad.

El presente trabajo tiene por objetivos encontrar la frecuencia de lesiones orales y la ubicación de éstas en pacientes VIH/SIDA de acuerdo a la edad y al sexo; además establecer la relación existente entre la cifra de linfocitos CD4 con las lesiones detectadas. El odontólogo debe conocer cada una de estas lesiones presentes en los pacientes infectados por VIH ya que muchas veces puede ser el primer profesional de la salud que detecte la enfermedad; además muchas de estas lesiones son indicadores clínicos de un deterioro en el sistema inmunológico. vellosa como las lesiones más comunes en
ISSN: 1560-9111

\section{ARTículo de RevisióN}

\section{Lorena Elvira Garibay Rodríguez', Luis \\ Cisneros Zárate}

CD. Práctica privada.

Departamento Académico Médico

Quirúrgico. Facultad de Odontología.

UNMSM

\section{Correspondencia:}

CD. Lorena Garibay Rodríguez

Condorama 112 - Higuereta - Surco

Teléfono: 449-2098

e-mail: legro74@yahoo.es

Palabras clave: Lesiones orales. Infección VIH. SIDA.

Key words: Oral lesions. VIH infection. AIDS.

\section{Materiales y método}

El trabajo se realizó en el Programa Preventivo Promocional Control ETS-VIH/SIDA del Hospital Guillermo Almenara Irigoyen (HNGAI). La muestra fue tomada de manera probabilística y estuvo constituida por 128 pacientes. Se eligieron pacientes que asistieron al HNGAI y presentaron positividad para el examen Western Blot y Elisa. Estos pacientes no debieron recibir medicación antifúngica (ketoconazol, fluconazol) ni tratamiento retroviral. Finalmente estos pacientes tuvieron que haber sido sometidos a un conteo de CD4 en un período de tiempo no mayor a 2 meses próximos al examen. Al paciente se le realizó un minucioso examen clínico, el método utilizado para la recolección de datos fue la observación y la información obtenida se registro en una ficha previamente diseñada.

\section{Resultados}

El trabajo fue realizado en 128 pacientes pertenecientes al Programa Preventivo Promocional Control ETS|VIH-SIDA del HNGAI que acudieron a la consulta médica. Del total de los pacientes estudiados 74 pertenecían al sexo masculino $(57,8 \%)$, mientras que 54 pertenecían al sexo femenino $(42,2 \%)$. En relación a la edad, el mayor número de pacientes se encontraba ubicado en el grupo de 26 a 35 años (43,8\%). El 53,1\% presentaba manifestaciones orales, mientras que el $46,9 \%$ no presentaban manifestaciones estomatológicas en el momento del examen. 
Cuadro 1. Distribución de las manifestaciones estomatólogicas en paciente VIH/SIDA - Hospital Nacional Guillermo Almenara Irigoyen. Lima - Perú 1999

\begin{tabular}{lcc}
\hline $\begin{array}{l}\text { Manifestaciones } \\
\text { Estomatológicas }\end{array}$ & $\mathbf{f}$ & $\%$ \\
\hline Fungicas & 38 & 29,7 \\
C. seudomembranosa & 28 & 21,9 \\
C. eritemtosa & 14 & 10,9 \\
Queilitis angular & 14 & 10.9 \\
Virales & 22 & 17,2 \\
H. zoster & 04 & 3,1 \\
H. simple & 07 & 5,5 \\
LOV & 09 & 7,0 \\
Molusco & 01 & 0,8 \\
Papiloma & 01 & 0,8 \\
Bacterianas & 05 & 4,0 \\
GUNA & 00 & 0,0 \\
Eritema gingival lineal & 05 & 4,0 \\
Autoinmunitaria, idiopatica & 18 & 14,1 \\
U. aftosa mayor & 11 & 8,6 \\
U. aftosa menor & 08 & 6,25 \\
Neeoplasia & 03 & 2,3 \\
Sarcoma de kaposi & 03 & 2,3 \\
\hline
\end{tabular}

\section{Discusión:}

El presente trabajo trata de describir la frecuencia de lesiones estomatológicas observadas al examen clínico en 128 pacientes infectados por $\mathrm{VIH}$, de los cuales el $57,8 \%$ pertenecen al sexo masculino y $42,2 \%$ a sexo femenino. Encontrándose que la razón hombre mujer fue de 1,37 a 1. Estos valores no concuerdan con los obtenidos con Field ${ }^{11}$ en su trabajo realizado en octubre de 1991 y enero de 1992 en el mismo hospital; el cual encontró una razón hombre mujer de 6,5 a 1. La diferencia probablemente se deba a la evolución misma de la epidemia pero los valores obtenidos son muy similares a los obtenidos por el PROCETTS, donde señala que la razón hombre-mujer en el año 1998 fue de 3 a 1;manifestando que para el año 2000 la razón será de 1 a 1 . Lo que indica que se ha producido un aumento en la transmisión heterosexual y por ende mayor incidencia de mujeres infectadas. ${ }^{12}$

De todas las manifestaciones estomatológicas encontradas el $29,7 \%$ de los pacientes presentan lesiones fúngicas. La candidiasis pseudomembranosa se presenta en el $21,9 \%$ de los pacientes siendo la zona más afectada la lengua, paladar blando, paladar duro y carrillo. Estos valores son muy similares a los obtenidos por Ramírez ${ }^{7}$ quien encontró que la candidiasis bucal representaba e $46 \%$ de las lesiones encontradas y el tipo pseudomembranoso se presentó en el 15\%, Field encontró también que la zona más afectada por candidiasis pseudomembranosa fue la lengua. ${ }^{11}$

El 57,1\% de los pacientes con candidiasis pseudomembranosa se encuentran en el grupo de recuento de CD4 de 0 a $199 \mathrm{cel} / \mathrm{mm} 3$, mientras que el $39,3 \%$ se ubicaba en el grupo de 200 a $499 \mathrm{cel} / \mathrm{mm} 3$. Aplicando la prueba de Chi cuadrado $(p<0,05 \%)$ se encontró que existía relación significativa entre la presencia de candidiasis pseudomembranosa y un nivel de CD4 por debajo de $200 \mathrm{cel} / \mathrm{mm} 3$ estos resutados coinciden con muchos autores que manifiestan que la infección micótica está estrechamente asociada con el deterioro inmunológico y es común encontrarla en pacientes con bajo conteo de CD4. ${ }^{6,13,14}$

La LOV se presentó en el $7 \%$ de pacientes
Cuadro 2. Distribución de las manifestaciones estomatólogicas en paciente VIH/SIDA según recuento $\mathrm{CD} 4 / \mathrm{mm}^{3}$ Hospital Nacional Guillermo Almenara Irigoyen. Lima - Perú 1999

\begin{tabular}{|c|c|c|c|c|c|c|}
\hline \multirow{2}{*}{$\begin{array}{l}\text { Manifestación } \\
\text { Estomatológica }\end{array}$} & \multicolumn{2}{|c|}{$\begin{array}{l}0-199 \\
\mathrm{~cd} 4 / \mathrm{mm}^{3} \\
n=28\end{array}$} & \multicolumn{2}{|c|}{$\begin{array}{l}200-499 \\
\mathrm{~cd} 4 / \mathrm{mm}^{3} \\
n=37\end{array}$} & \multicolumn{2}{|c|}{$\begin{array}{l}500 \text { a mas } \\
\mathrm{cd} 4 / \mathrm{mm}^{3} \\
\mathrm{n}=03\end{array}$} \\
\hline & $f$ & $\%$ & $f$ & $\%$ & $f$ & $\%$ \\
\hline Candidiasis seudomembranosa & 16 & 57,0 & 11 & 39,3 & 01 & 03,6 \\
\hline Candidiasis eritemtosa & 04 & 28,5 & 10 & 71,4 & 00 & 00,0 \\
\hline Queilitis angular & 08 & 57,1 & 06 & 42,9 & 00 & 00,0 \\
\hline Herpes zoster & 01 & 25,0 & 02 & 50,0 & 01 & 25,0 \\
\hline Herpes simple & 02 & 28,6 & 05 & 71,4 & 00 & 00,0 \\
\hline Leucoplasia oral vellosa & 07 & 77,8 & 02 & 22,2 & 00 & 00,0 \\
\hline Molusco & 00 & 00,0 & 01 & 100 & 00 & 00,0 \\
\hline Ulcera aftosa & 05 & 27,8 & 11 & 61,1 & 02 & 11,1 \\
\hline Eritema gingival lineal & 02 & 40,0 & 03 & 60,0 & 00 & 00,0 \\
\hline Sarcoma de kaposi & 01 & 33,3 & 02 & 66,7 & 00 & 00,0 \\
\hline Papiloma & 00 & 00,0 & 01 & 100 & 00 & 00,0 \\
\hline
\end{tabular}

$p<0,05(3,84 \%) \quad x^{2}$ candidiasis seudomembranosa: 4,96 (significativo) $x^{2}$ leucoplasia oral vellosa: 4,95 (significativo) estudiados, esta lesión no había sido descrita antes de la epidemia del SIDA pero también puede encontrarse en otras formas de inmunosupresión. Casi todos los pacientes con LOV pueden desarrollar hacia el SIDA en 24 meses y fallecer en un tiempo promedio de 44 meses. $^{8}$ Suele aparecer tarde en la evolución de la enfermedad, generalmente 5 años después del contagio cuando ya existe un importante grado de inmunosupresión. ${ }^{9,15}$

Al aplicar la prueba de Chi2, se encontró que sí existía relación entre la LOV y un recuento de CD4 por debajo de $200 \mathrm{cel} / \mathrm{mm} 3$ ( $\mathrm{p}<0,05 \%$ ) con un grado de libertad de 1 . Estos datos concuerdan con los obtenidos por Ramírez ${ }^{7}$, Delgado ${ }^{10}$ y Field ${ }^{11}$, los cuales manifestaron que esta lesión es más frecuente en estadios avanzados de la enfermedad.

Al término de la investigación se llega a las siguientes conclusiones:

1. La frecuencia de pacientes HIV-SIDA según el sexo fue de $42,2 \%$ para las mujeres y $57,8 \%$ para los varones.

2. El $43,8 \%$ de de los pacientes se encontraban entre 26 y 35 años.

3. Las manifestaciones estomatológicas más frecuentes fueron candidiasis pseudomembranosa $29,7 \%$ (lengua, paladar blando, paladar duro y carrillo), úlceras aftosas 14,1\% (carrillo, lengua y labio), la candidiasis eritematosa $10,1 \%$ (paladar blando) queilitis angular $10,1 \%$ y la leucoplasia oral vellosa $7 \%$.

4. La disminución de CD4 favorece la aparición de una variedad de lesiones orales principalmente la candidiasis pseudomembranosa y la leucoplasia oral vellosa considerándolas como indicadores de deterioro inmunológico.

\section{Referencias bibliográficas:}

1. FARRERAS V.Medicina Interna.XII Edición. Madrid España: Mosby-Doyma Libros S.A. 1997.

2. LIFSON A.R. Oral lesions and epidemiology of HIV. En Greenspan J.S.,Greenspan D. editores. Oral manifestations of HIV infection: Proceedings of 2nd Internacional workshop on the oral manifestations of HIV infection. Jan 31st - Feb 3rd 1993. Carol Stream ILL: Quittesence Publishing 1995.
3. GREENSPAN D. Opportunistic Infections of the mouth. The AIDS Knowledge Base (5.2)Jan. 1994.

4. SCHMIDT - WESTHAUSEN A., GRUNEWALD T., REICHART P.A.and POHLE H.D.Oral manifestations in 70 german HIV infected women. Oral Dis. England. Suppl. 1997;1:18-27.

5. GREENSPAN J.S. Sentinels and Signposts The epidemiology and significance of the oral manifestations of HIV disease. Oral Dis. England 1997; 3 Suppl 1:13-17.

6. BURKET. Medicina Bucal. Diagnóstico y Tratamiento. 8va ed. México: Nueva Editorial Interamericana.1986.

7. RAMíREZ V., y col. Patología Bucal en 161 pacientes VIH positivos asintomático y sintomáticos. Rev.Invest.Clin. 1992;44(1):43-51.

8. GREENSPAN D. y GREENSPAN J.S. Ora complication of HIV infection. The medical management of AIDS. Merle A.Sande, Paul A. Volberding, Fifth edition. United States of America: WB Saunders Company. 1997.

9. MUÑOZ PEREZ M.A. Manifestaciones orales del SIDA. Monografías de Dermatología. 1996:9:110-118.

10.DELGADO A.W. Manifestaciones Orales de la infección por VIH/SIDA. De SIDA Epidemiología, Diagnóstico, Tratamiento y Control de la infección VIH/SIDA. OMS/OPS Ministerio de Salud. Asociación Vía LibreHIVOS, Perú: Fondo Editorial 1994.

11.FIELD J.M. Frecuencia de lesiones orales clínicamente detectables en tejidos blandos móviles de individuos infectados por el virus del HIV en el Hospital Guillermo Almenara Irigoyen. Tesis para optar el grado de Cirujano Dentista. UPCH. Fac. de Estomatología. 1992.

12. Programa de Control de Enfermedades de Transmisión Sexual y SIDA (PROCETTS) de Ministerio de Salud. Magnitud de la Infección por VIH. Internet: www.procetts.com.pe 1999.

13. DELGADO A.W. Infección por el VIH/ SIDA en Estomatología. Odontología Peruana 1992; 6(8). 19-28.

14. CEBALLOS S.A. y DELGADO A.W. Micosis Bucales.Grupo Madrid: Aula Médica S.A. 1996.

Recibido : 23-04-2007

Aceptado para publicación: 25-05-2007 